\title{
Studies of Chaotic Transport of Electrons in Quantum Boxes
}

\author{
D. K. FERRY and G. EDWARDS \\ Department of Electrical Engineering, Arizona State University, Tempe, AZ 85287-5706
}

\begin{abstract}
Recent studies of transport through ballistic quantum dot resonators have revealed a complex array of behavior, including the existence of "universal" conductance fluctuations which are presumed to arise from the chaotic behavior of the underlying classical dynamics. In this paper, the results of studies on the classical ballistic transport of carriers, within a quasi-twodimensional electron gas, through a 1.0 micron square structure in a magnetic field are presented.
\end{abstract}

Recently, interest has focused upon the study of transport in small quantum devices, formed in modulationdoped GaAs/AlGaAs heterostructures, in which the transport is characterized as ballistic, as the mean-free path of the carriers is larger than the characteristic dimensions of a quantum dot formed between two quantum point contacts (QPCs). These quantum structures show conductance fluctuations (for small variations in the magnetic field or gate voltage) and weak localization [1-4], two properties that are normally connected with diffusive transport in systems of small mean-free path. It has been suggested that the fluctuations and weak localization are the result of quantum transport corresponding to semi-classical chaos within the dot, and the study of such quantum dots is thought to probe the transition to quantum chaos $[5,6]$.

In this paper, we study the classical billiard transport of particles through a nanoscaled quantum dot formed by a split-gate with two QPCs as entry and exit ports. The structure is nominally a $1.0 \mu \mathrm{m}$ square cavity (inset to Fig. 1) with an inlet and exit port on opposite sides. The effect of a Sinai diffuser, added to the side spanning between the two ports, is to mimic the electrostatic profile. It is normally thought that a (closed) square cavity is regular, and not chaotic, but there is evidence that the existence of the two ports, and the resulting spread in input angles, allows for the onset of chaos [6]. The density in the electron gas is $4.4 \times 10^{11} \mathrm{~cm}^{-2}$, in keeping with the experiments of Bird et al. [4]. We compute the areal distribution functions, and Poincare sections for transport in the dot. Finally, we compare the calculated correlation function with that calculated by a recursive quantum Green's function, and with the experiments of ref. [4].

It may be assumed that the fluctuations arise from the complex scattering dynamics, which leads to phase interference in the quantum case. The important point is that the characteristic correlation scale in the magnetic field, or the Fermi energy, for the fluctuations in the quantum transport can be predicted from properties of the equivalent classical orbits in the same structure [5]. The swept orbit path area in a magnetic field from the vector potential is

\footnotetext{
* Work supported in part by the Office of Naval Research, and by NEDO under the International Joint Program "Nanostructures and Electron Waves Project"
} 


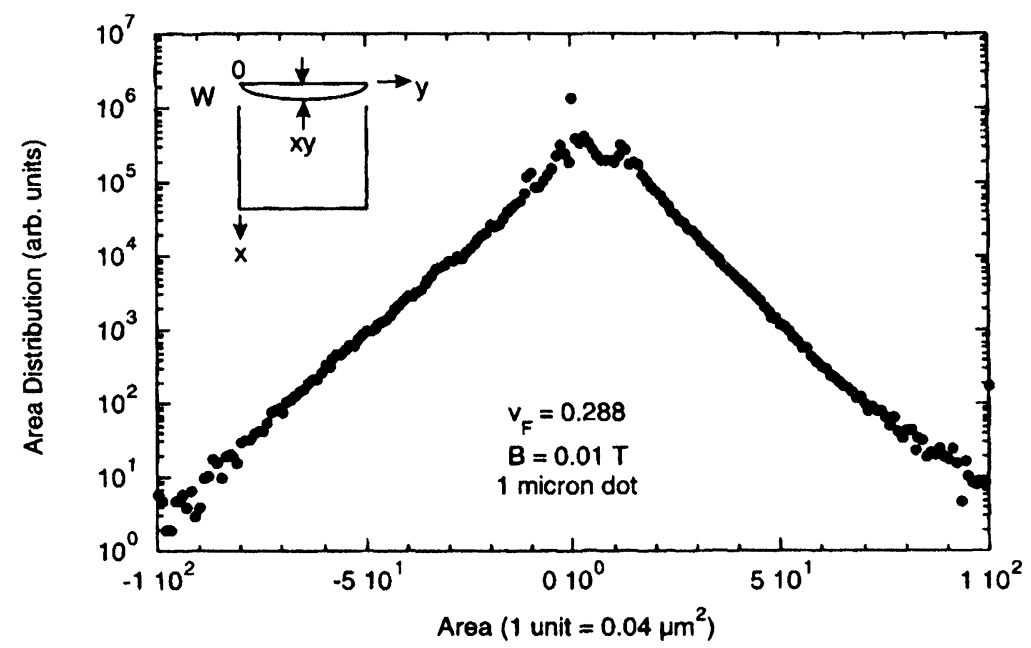

FIGURE 1 The area distribution function for the classical ballistic transport through the square quantum dot. The inset depicts the structure of the dot

$$
\begin{aligned}
S=\frac{e}{\hbar B} \int A \cdot d l \rightarrow f(S) \sim \exp \left[-2 \pi \alpha_{c l}|S|\right] & \\
& \alpha_{c l}^{-1} \approx \frac{h}{2 \pi e \Delta B},
\end{aligned}
$$

where $\Delta B$ is the difference in field encountered in the magnetic correlation function, and $\alpha_{c l}$ is defined by the exponentially distributed area distribution function $f(S)$. That is, $\alpha_{\mathrm{cl}}{ }^{-1}$ gives the rms area enclosed by typical trajectories in the structure. The exponential decay of the area distribution function leads to a Lorentzian-squared behavior for the conductance fluctuation correlation function, as

$$
F(\Delta B)=\left|\int_{\infty}^{\infty} f(S) e^{i e \Delta B S / \hbar} d S\right|^{2}=\frac{F(0)}{\left[1+\left(\frac{e \Delta B}{\alpha_{c l} h}\right)^{2}\right]^{2}}
$$

In Fig. 1, $f(S)$ for the quantum dot studied here is plotted for $W=0.1 \mu \mathrm{m}$ and $x y=0.1 \mu \mathrm{m}$. A total of $9 \times 10^{6}$ particles are introduced from the inlet port (left). The Poincare sections are computed by plotting the position $\mathrm{y}$ and velocity $v_{y}$ for particles which hit the transverse wall opposite the diffuser $(x=1 \mu \mathrm{m})$. The $\mathrm{y}$-axis is along the diffuser from the inlet port to the outlet port $(x=0)$, and the $x$-axis is downward in the inset to Fig. 1. These sections are shown in Fig. 2 for three different conditions. In panel (a), we plot the case for which the incoming particles enter with a narrow spread around $\theta=\pi / 3$, where $\theta$ is measured from the positive $x$-axis, $W=0.01 \mu \mathrm{m}$, and no diffuser is used. Clearly in this case, the trajectories are regular with no chaotic activity appearing. The gray scale imaging allows us to also plot the magnitude of

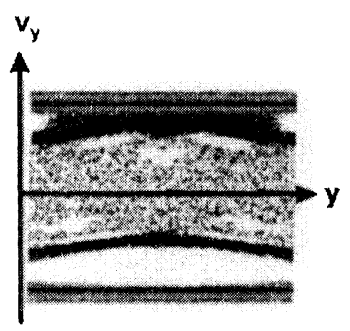

(a)

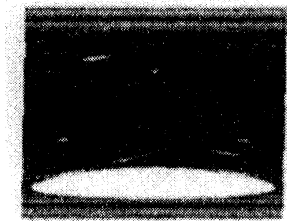

(b)

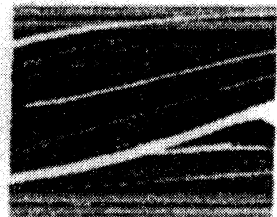

(c)

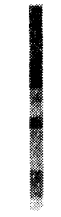

$|v x|$

FIGURE 2 Poincare sections for the trajectories for a variety of situations (discussed in the text) 


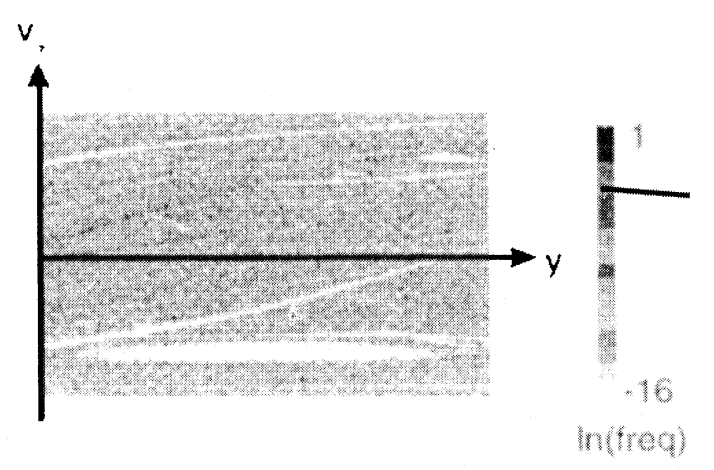

FIGURE 3 The Poincare frequency map for the section of Fig. 2 (c)

$v_{x}$ as gray intensity. In panel (b), the incoming particles are uniformly distributed in $\theta$ across the inlet port, $W=0.05 \mu \mathrm{m}$, and no diffuser is used. It is apparent that the broad distribution of incoming trajectories in the angle accounts for considerable filling of the phase space. Finally, in panel (c), a broad incoming distribution is used with $W=x y=0.1 \mu \mathrm{m}$. Clearly, here the phase space is nearly fully occupied, but there remain "scratches" of unoccupied phase space, whose interpretation is not fully understood at this time.
While the Poincare sections allow us to determine which points in the phase space projection are actually visited by ballistic orbits, it cannot tell us how often these points are visited. For this, we construct a histogram, which is a Poincare frequency plot, as shown in Fig. 3. The gray scale is used to indicate the logarithm of the frequency at which each point in phase space is visited. The uniformity of this plot, corresponding to a broad incoming beam ( $W=0.05$ $\mu \mathrm{m}$ and $x y=0.025 \mu \mathrm{m}$ ), gives an indication of the manner in which the particles sweep through the phase space in the chaotic environment.

Finally, in Fig. 4, the magnetic field correlation function obtained from Fig. 1 and eqn. (2) is plotted and compared to the equivalent function determined from a fully quantum mechanical simulation of the same structure with recursive Green's functions [7], and with the experimental results of ref. [4]. It is clear that the two theoretical approaches give quite comparable results, and the quantum mechanical fluctuations are clearly related to the classical chaotic behavior of the cavity. However, the experimental curve does not decay quite as rapidly, but this is easily understood. Although the quantum dot is lithographically defined as a $1.0 \mu \mathrm{m}$ square, the depletion around

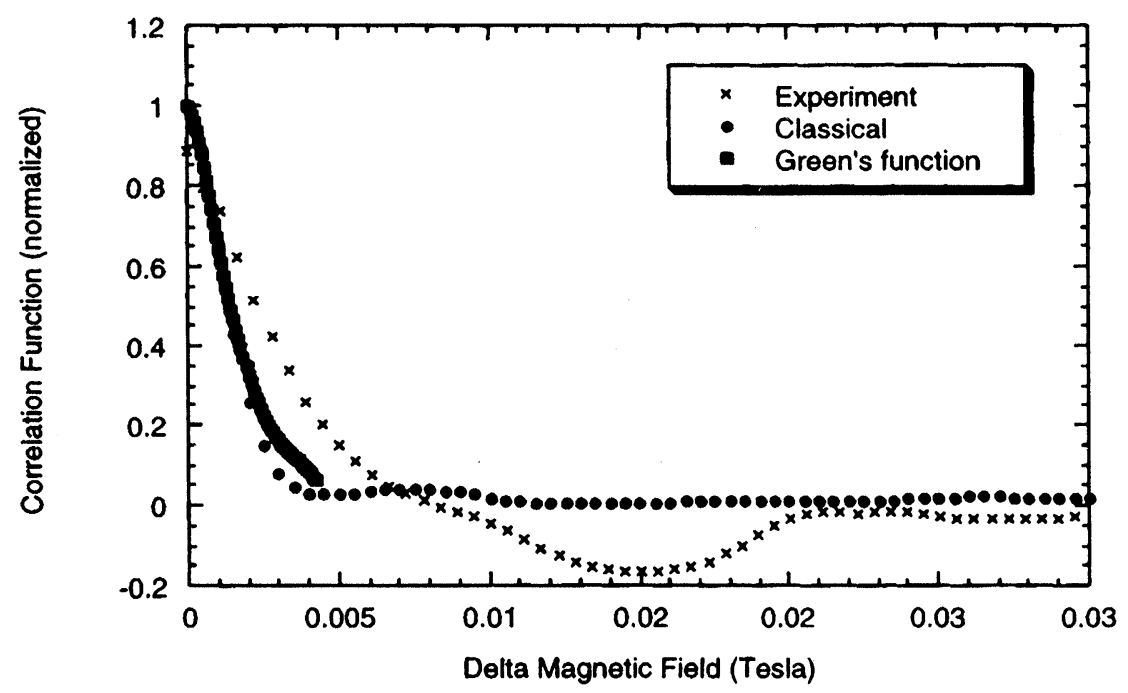

FIGURE 4 Magnetic field correlation function computed from the classical area distribution function $(\bullet)$, a recursive Green's function quantum computation ( $\mathbf{\square})$, and the experimental curve obtained in a comparably-sized dot $(\times)$ from ref. [4] 
the gate edges reduces the size of the actual active area. Based upon measurements of Aharonov-Bohm oscillations at higher magnetic fields, it is estimated that the actual active area is only $0.64 \mu \mathrm{m}^{2}$. From (3), this should increase the value of $\Delta B$ from $1.4 \mathrm{mT}$ to $2.18 \mathrm{mT}$, which is to be compared with the observed value of $2.6 \mathrm{mT}$, which is reasonably close for the uncertainty in the experiment (and in the theory). In fact, at these lower magnetic fields, it is likely that the actual active area is somewhat smaller than the size determined at higher magnetic fields, which would improve the agreement.

In summary, we have carried out numerical simulations of the classical transport of ballistic particles in a nanoscaled quantum dot. The results are shown to agree with recursive Green's functions and experiment in the chaotic regime.

\section{References}

[1] G. Timp, H. U. Baranger, P. de Vegvar, J. E. Cunningham, R. E. Howard, R. Behringer, and P. M. Mankiewich, Phys. Rev. Lett. 59, 3011 (1988).
[2] A. M. Chang, T. Y. Chang, and H. U. Baranger, Phys. Rev. Lett. 63, 996 (1989).

[3] C. M. Markus, A. J. Rimberg, R. M. Westervelt, P. F. Hopkins, and A. C. Gossard, Phys. Rev. Lett. 69, 506 (1992).

[4] J. P. Bird, K. Ishibashi, D. K. Ferry, Y. Ochiai, Y. Aoyagi, and T. Sugano, Phys. Rev. B 51, 18037 (1995).

[5] R. A. Jalabert, H. U. Baranger, and A. D. Stone, Phys. Rev. Lett. 65, 2442 (1990).

[6] H. U. Baranger, R. A. Jalabert, and A. D. Stone, Phys. Rev. Lett. 70, 3876 (1993).

[7] G. Edwards, A. Grincwagj, and D. K. Ferry, Surf. Sci., in press.

\section{Biographies}

David K. Ferry is Regents' Professor of Electrical Engineering at Arizona State University, where he pursues research in quantum transport in mesoscopic devices.

Gerard Edwards is a postdoctoral research scientist at ASU. He received his $\mathrm{Ph}$. D. from Exeter in 1992, following which he spent two years at Nottingham as a postdoctoral fellow. 

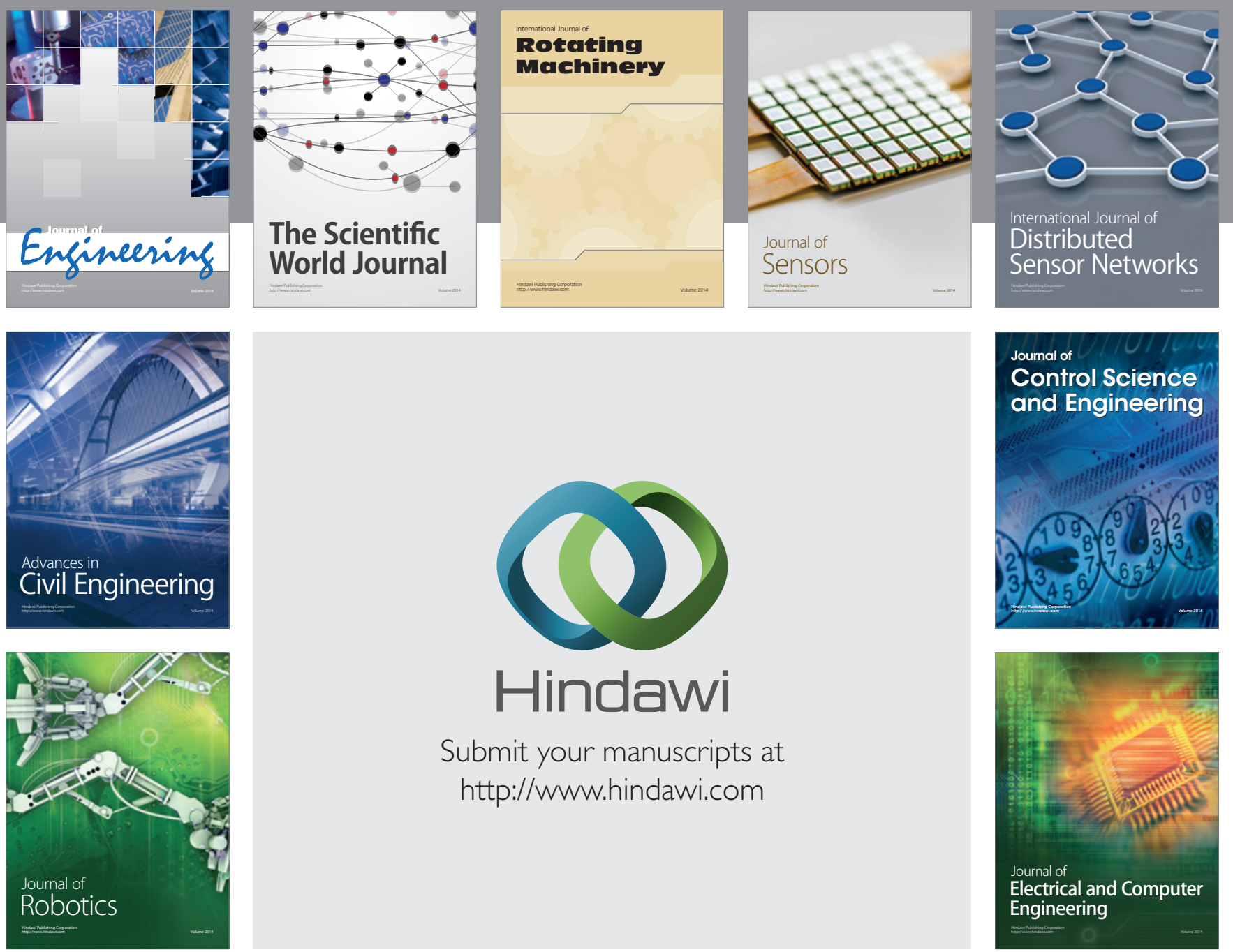

Submit your manuscripts at

http://www.hindawi.com
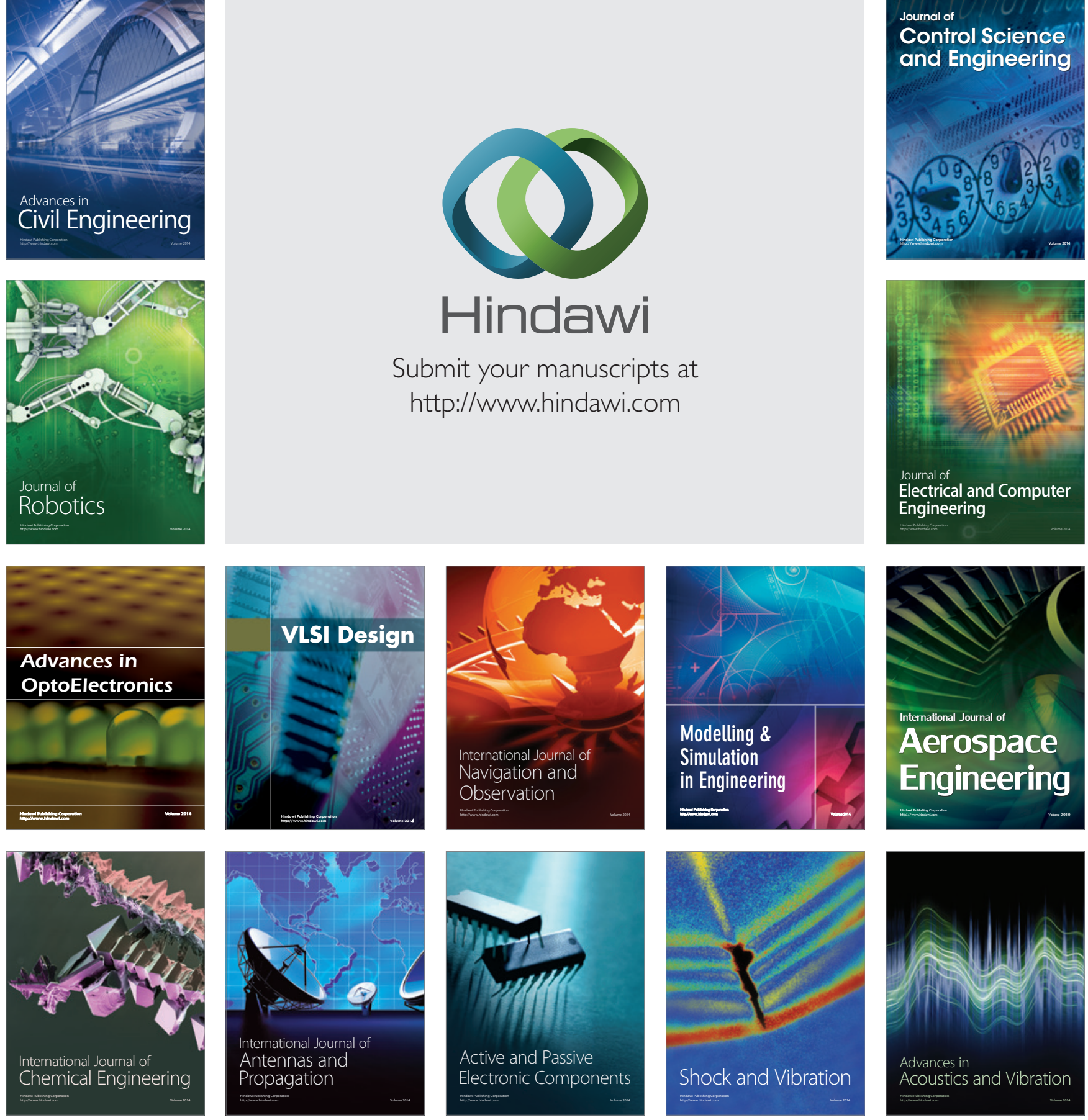